\title{
First Degree Relatives of Bangladeshi Prediabetic Subjects are at Increased Risk for Developing Glucose Intolerance
}

\author{
SM SHEFIN ${ }^{\mathrm{a}}$, MO FARUQUE ${ }^{\mathrm{b}}$, SH KHANDKER ${ }^{\mathrm{c}}, \mathrm{ZA} \mathrm{LATIF}^{\mathrm{d}}, \mathrm{L} \mathrm{ALI}^{\mathrm{e}}$
}

Summary:

Background: The racial variation in genetic susceptibility of Type2 diabetes mellitus is well established. The stages of impaired fasting glucose (IFG) and/or Impaired glucose tolerance (IGT) (collectively known as 'prediabetic stages) are combined to be intermediate in the natural history of diabetes, but their genetic susceptibility are still a matter of investigation. Family study is the primary step to explore genetic susceptibility. In particular, there has been no study in Bangladesh related to genetics of prediabetes.

Objectives: The present study aimed to explore the genetic susceptibility of prediabetes in Bangladeshi population by observing the clustering of dysglycemia in first degree relatives of prediabetes.

Methodology: The study was designed as an experimental group comparison study. Newly detected prediabetic subjects (isolated IFG, IGT, IFG+IGT) were collected from BIRDEM OPD and reconfirmed by OGTT following WHO guidelines and sub grouped.

Relatives of the prediabetes, up to first generation, were included as cases and termed as R-IFG (first degree relatives of IFG), R-IGT (first degree relatives of IGT), R-IFG-IGT (first degree relatives of IFG-IGT) corresponding to the

a. Dr. Sultana Marufa Shefin, MD (EM), Dept of Endocrinology and Diabetology, BIRDEM, Dhaka

b. Md Omar Faruque, PhD, Dept of Biochemistry and Cell Biology, BIRDEM, Dhaka

c. Dr. Sazzad Hossain Khandker, DO (Opth), Dept. of Ophthalmology, BIRDEM, Dhaka

d. Prof. Zafar Ahmed Latif, FCPS (Med), Dept of Endocrinology and Diabetology, BIRDEM, Dhaka

e. Prof. Liaquat Ali, PhD, Director, Bangladesh Institute of Health Sciences, Dhaka

Address of Correspondence: Dr. Sultana Marufa Shefin, MD (EM), Registrar, Dept of Endocrinology and Diabetology, BIRDEM, Dhaka, Email: shefin_neon@yahoo.com

Received: 24 April, 2010

Accepted: 5 December, 2010 subgroups of prediabetes. Each relative underwent an OGTT following the same guideline.

Blood glucose was measured by glucose oxidase method.

Results: Different types of prediabetic subjects (IFG IGT, $I F G+I G T)$ and their first degree relatives ( $R \_I F G, R \_I G T$, $\left.R \_I F G+I G T\right)$ were studied. Among 41 first degree relatives of IFG (R_IFG), 2 (4.9\%) had IFG 4 (9.8\%) had IGT, 1 (2.4\%) had combined IFG+IGT, 5 (12.5\%) had T2 DM and 29 (70.7\%) had normoglycemia. Among 116 first degree relatives of IGT (R_IGT) none (0.00\%) had IFG 15 (12.9\%) had IGT, 2 (1.7\%) had combined IFG+IGT, 22 (19\%) had diabetes and 77 (66.4\%) having absolutely normal OGTT reports. Among 76 first degree relatives of IFG+IGT (R_IFG+IGT), 2 (2.6\%) had IFG, 4 (5.3\%) had IGT, 1 (1.3\%) had combined IFG+IGT, 19 (25\%) had diabetes and 50 (65.8\%) were normoglycemic.

Conclusion: Clustering of pre-diabetes and diabetes is present in families of prediabetic subjects and they should be taken as a major target for primary prevention of these disorders.

Key words: Prediabetes, IFG, IGT, IFG+IGT, First degree relatives, Glucose intolerance.

(J Bangladesh Coll Phys Surg 2011; 29: 21-26)

Introduction:

Diabetes is by far the commonest endocrine disorder in the wo-rld. The prevalence of diabetes (20-79 years) in South--East Asia in 2003 was 5.6\% and predicted to be $7.5 \%$ in 2025 and in Bangladesh 3.9\% in 2003 \& predicted to be $4.8 \%$ in $2025^{1}$.Asian Indians (like people from India, Pakistan, Bangladesh and Sri Lanka) have surprisingly high prevalence of type 2 diabetes compared to Caucasians. Excessive insulin resistance in Asian Indians compared to Caucasians may be one of the contributing factors. This difference in the degree of insulin resistance may be explained by either an environmental or genetic factors or by combination of both $^{2-9}$. All forms of diabetes can pass through a stage 
of IFG and/or IGT. In a study on Asian ethnicity, it was found that impaired glucose tolerance was more prevalent than impaired fasting glycemia in all Asian populations studied for all age-groups ${ }^{10}$. The rising prevalence rate of IGT may be mainly due to diabetogenic lifestyle factors that lead to obesity and increasing life expectancy. The rising prevalence of IGT is assumed to increase from 8.2 to $9.0 \%$ worldwide and 7.1 to $7.8 \%$ in Bangladesh from 2003 to 2025 in adults (20-79 yrs age groups) ${ }^{1}$. The crude prevalence of IFG was $12.4 \%$ in rural population of Bangladesh; the agestandardized prevalence of IFG was $13.0 \%{ }^{11}$. Approximately $50 \%$ of people with IFG have IGT and $20-30 \%$ with IGT also has IFG ${ }^{11}$. About $25 \%$ of people with IGT progress to type 2 diabetes within 5 years, while the majority either remain within that category (50\%) or revert to normal glucose tolerance (25\%). Interestingly, there is a tendency for the prevalence rates of IGT to decline as those of type 2 diabetes mellitus (DM) rise, perhaps suggesting that areas with a high ratio of IGT:Type $2 \mathrm{DM}$ are at an earlier stage of type 2 DM epidemic and thus a particular target for preventive strategies $^{12}$.

IFG and IGT are asymptomatic and unassociated with any manifested morbidity, but their sole significance lies in the fact that they predict future diabetes or cardiovascular diseases ${ }^{13}$. The main features of IFG/ IGT are: 1) a stage in the natural history of disordered glucose metabolism, 2) can lead to any type of diabetes, 3) increased risk of progression to diabetes, 4) some patients may revert to normoglycemia. Both IFG and IGT are similarly associated with an increased risk of DM. Risk is higher where IGT and IFG coexists $^{15}$. IFG/ IGT is often associated with "Metabolic Syndrome", which includes obesity (specially abdominal or visceral obesity), dyslipidemia (high triglyceride level, low HDL level and smaller LDL particle diameter) and hypertension, along with glucose intolerance (type 2 diabetes mellitus, IGT or IFG) and hyperinsulinemia. Evidence is accumulating that insulin resistance may be the common etiological factor for the individual components of the metabolic syndrome. Both insulin resistance and impaired b-cell function are important pathophysiologic changes contributing to the onset and development of type 2 diabetes. It probably results from a genetically determined reduction in insulin sensitivity, compounded by exposure to the environmental factors, which further impair insulin action. A striking feature of type 2 diabetes is the strength of its genetic component. In a study suggest a more pronounced effect of a family history of diabetes on risk of type 2 diabetes in men and women. While both a family history of diabetes and lifestyle risk factors had effects on type 2 diabetes, irrespective of sex, these effects did not appear to interact ${ }^{16}$.

Apart from genes, families share environments, culture and habits, yet familial aggregation of the disease is another source of evidence for a genetic contribution to the disease. Abnormal glucose tolerance is common in first-degree relatives of type 2 Diabetes Mellitus patients; but first degree relatives of prediabetes are not studied yet. Impairment of insulin secretory capacity and / or insulin sensitivity is present among prediabetes subjects. So the present study is designed to detect glycemic status of first degree relatives of prediabetes subjects in search of genetic linkage of T2DM.

\section{Subject and methods}

The study was conducted in the Dept of Endocrinology and Diabetology and Biomedical Research Group, Research Division, Bangladesh Institute of Research and Rehabilitation in Diabetes, Endocrine and Metabolic Disorders (BIRDEM), Dhaka, Bangladesh, during the period of March 2006 - April 2007. It was a cross sectional study. The subjects were selected purposively. Total 303 subjects were included in the study, 41 first degree relatives (RIFG group) of newly detected 14 isolated IFG cases, 116 first degree relatives (RIGT group) of newly detected 36 isolated IGT cases and 76 first degree relatives (RIFG-IGT group) of 20 newly detected combined IFG-IGT cases were included in the study. Subjects of extreme of age $(<10$ years \& $>65$ years), those with serious comorbid diseases (infection, stroke, myocardial infarction, major surgery, malabsorption, CLD, renal failure etc.), with history of using drugs significantly affecting glucose metabolism (glucocorticoids, phenytoin, estrogen, progesterone, testosterone, thiazide diuretics etc.) or pre-existing diabetes, were excluded from the study.

\section{Collection of the subjects}

Subjects were selected from the Out-Patient Department (OPD) of BIRDEM who diagnosed as prediabetes first 
time, (Newly detected IFG subjects (Fasting serum glucose 6.1- $6.9 \mathrm{mmol} / \mathrm{L}$ and serum glucose 2 hour after 75 gm oral glucose $<7.8 \mathrm{mmol} / \mathrm{L}$ ), newly detected IGT subjects (Fasting serum glucose $<6.1 \mathrm{mmol} / \mathrm{L}$ and serum glucose 2 hour after 75 gm oral glucose $7.8-11.0 \mathrm{mmol} /$ L), newly detected combined IFG-IGT subjects (Fasting serum glucose 6.1- $6.9 \mathrm{mmol} / \mathrm{L}$ and serum glucose 2 hour after 75 gm oral glucose 7.8-11 mmol/L).

After taking brief history, the purpose of the study was explained in details to each subject. They were advised to take unrestricted carbohydrate diet, to do normal physical activities and to avoid drugs that significantly interfere with blood glucose level (like Glucocorticoids, oral contraceptives containing Levonorgestrel or high-dose estrogen, Phenytoin, highdose Thiazide diuretics, etc.) for 3 days. They were also advised to abandon the program if they became sick. Then they were requested to report to Biomedical Research Group, Research Division, BIRDEM after 3 days at morning following an overnight (8-14 hours) fasting. When the subjects reported, informed written consent was taken and OGTT was done to reconfirm prediabetic state. After reconfirmation the subject was requested to bring their first-degree relatives at least of two generation, mentioning all the above prerequisites. Then sample was collected and printed questionnaire was filled up.

The subjects were selected purposively. About 1200 patient were interviewed at OPD, BIRDEM detected as a new case of IGR (IFG, IGT and combined IFGIGT). Among them 410 IGR subjects were enlisted their name to recheck their IGR status at BMRG laboratory according to WHO criteria, but only 160 was reported, and they were reconfirmed as 22 IFG, 76 IGT, 38 IFGIGT, 12 DM and 12 normal OGTT subjects. The IGR subjects were requested to bring their first degree relatives, at least 3 in number and if possible from two generations among parent, sibling and offspring. Finally 41 first degree relatives (RIFG group) of 14 isolated IFG subjects, 116 first degree relatives (RIGT group) of 36 isolated IGT subjects and 76 first degree relatives (RIFG-IGT group) of 20 combined IFG-IGT subjects were included in this study.

\section{Analytical methods and lab analysis}

Serum glucose (fasting and 2 hours after $75 \mathrm{~g}$ glucose) was measured by Glucose-Oxidase (GOD-PAP) method (Randox Laboratories Ltd., UK).

Statistical analysis

Statistical analysis was performed using SPSS (Statistical Package for Social Science) software for Windows version 10 (SPSS Inc., Chicago, Illinois, USA).

\section{Results:}

In this cross sectional study different types of newly diagnosed prediabetic subjects (IFG, IGT, IFG+IGT) and their first degree relatives (R_IFG, R_IGT, R_IFG+IGT) were studied. Total 303 subjects were included in the study, 41 first degree relatives (RIFG group) of 14 isolated IFG cases, 116 first degree relatives (RIGT group) of 36 isolated IGT cases and 76 first degree relatives (RIFG-IGT group) of 20 combined IFG-IGT cases. Among them 129 (42.57\%) were male and 174 (57.43\%) were female. Ages of prediabetes subjects (IFG, IGT, IFG+IGT) were with in $4^{\text {th }}$ decade and of their first degree relatives were (R_IFG, R_IGT, R_IFG+IGT) were in $3^{\text {rd }}$ decade. (Table I). Among the study population 213 (70.30\%) resides in urban area, 35 (11.55\%) in semi urban area and 55 (18.15\%) in rural area. (Table I) Among 41 first degree relatives of IFG (R_IFG), 2 (4.9\%) have IFG, 4 (9.8\%) have IGT, 1 (2.4\%) has combined IFG+IGT, 5 (12.5\%) have diabetes and 29 (70.7\%) having absolutely normal OGTT reports. Among 116 first degree relatives of IGT (R_IGT), none (0.00\%) have IFG, 15 (12.9\%) have IGT, 2 (1.7\%) have combined IFG+IGT, 22 (19\%) have diabetes and 77 (66.4\%) having absolutely normal OGTT reports. Among 76 first degree relatives of IFG+IGT (R_IFG+IGT), 2 (2.6\%) have IFG, 4 (5.3\%) have IGT, 1 (1.3\%) has combined IFG+IGT, 19 (25\%) have diabetes and 50 (65.8\%) having absolutely normal OGTT reports. (Table II). Considering IFG, IGT, IFG+IGT and Diabetes as abnormal OGTT group among the 303 study subjects including both prediabetes and their first degree relatives found that $66.7 \%$ of them were from urban residence, $14.3 \%$ from semi urban residence and $19 \%$ from rural communities (Table III). 


\section{Table I}

Age, sex and residence status of the study subjects

\begin{tabular}{|c|c|c|c|c|c|c|c|}
\hline \multirow[t]{2}{*}{ Group } & \multirow[t]{2}{*}{ No. } & \multirow[t]{2}{*}{ Age, yrs } & \multicolumn{2}{|c|}{ Sex } & \multicolumn{3}{|c|}{ Residence } \\
\hline & & & Male N (\%) & Female N (\%) & Urban No (\%) & Semiurban No (\%) & Rural No (\%) \\
\hline IFG & 14 & $45 \pm 21$ & $8(57.1)$ & 6 (42.9) & $10(71.4)$ & $1(7.1)$ & $3(21.4)$ \\
\hline IGT & 36 & $41 \pm 12$ & $8(22.1)$ & $28(77.8)$ & 23 (63.9) & 5 (13.9) & $8(22.2)$ \\
\hline IFG+IGT & 20 & $45 \pm 10$ & $7(35)$ & $13(65)$ & $17(85)$ & $1(5)$ & $2(10)$ \\
\hline R_IFG & 41 & $36 \pm 16$ & 18 (43.9) & $23(56.1)$ & $28(68.3)$ & $3(7.3)$ & $10(24.4)$ \\
\hline R_IGT & 116 & $36 \pm 18$ & $50(43.1)$ & 66 (56.9) & 77 (66.4) & $18(15.5)$ & $21(18.1)$ \\
\hline R_IFG+IGT & 76 & $35 \pm 17$ & $38(50)$ & $38(50)$ & $58(76.3)$ & $7(9.2)$ & $11(14.5)$ \\
\hline Total & 303 & & $129(42.6)$ & $174(57.43)$ & 213(70.30) & 35 (11.55) & 55 (18.15) \\
\hline
\end{tabular}

IFG, impaired fasting glucose; IGT, impaired glucose tolerance; IFG+IGT, combined IFG \& IGT; R_IFG, First degree relatives of IFG; R_IGT, First degree relatives of IGT; R_IFG+IGT, First degree relatives of IFG+IGT.

\section{Table II}

Pattern of Glycemic status among the first degree relatives of different prediabetic subjects

\begin{tabular}{lcccccc} 
Group & Normal OGTT & \multicolumn{4}{c}{ Abnormal OGTT } \\
& Normal N (\%) & IFG N (\%) & IGT N (\%) & IFG+IGT N (\%) & DM N (\%) & Total, N (\%) \\
\hline R_IFG, n=41 & $29(70.7)$ & $2(4.9)$ & $4(9.8)$ & $1(2.4)$ & $5(12.2)$ & $12(29.3)$ \\
R_IGT, n=116 & $77(66.4)$ & 0 & $15(12.9)$ & $2(1.7)$ & $22(19)$ & $39(33.6)$ \\
R_IFG-IGT, n=76 & $50(65.8)$ & $2(2.6)$ & $4(5.3)$ & $1(1.3)$ & $19(25)$ & $26(34.2)$ \\
\hline
\end{tabular}

DM, diabetes mellitus; OGTT, oral glucose tolerance test.

Table-III

Area of residences of total study subjects characterized as having normal OGTT and abnormal OGTT

\begin{tabular}{lcccc} 
Group & Total, N (\%) & Urban, N(\%) & Semiurban, N(\%) & Rural, N(\%) \\
\hline Abnormal OGTT & $147(100)$ & $98(66.7)$ & $21(14.3)$ & $28(19)$ \\
Normal OGTT & $156(100)$ & $115(73.7)$ & $14(9.0)$ & $27(17.3)$ \\
\hline
\end{tabular}

\section{Discussion:}

Diabetes mellitus is distributed world wide mostly type 2, without any sex difference. Risk of type 2 diabetes increases after 40 years of age but happened at earlier age due to change of life style and dietary habit, less physical activity and weight gain. In this study 129 (42.57\%) were male and 174 (57.43\%) were female subjects. Age of different prediabetes were in same decade ( $4^{\text {th }}$ decade) and that of their relatives were match within their group ( $3^{\text {rd }}$ decade). Among the study population 213 (70.30\%) resides in urban area, 35
(11.55\%) in semi urban area and 55 (18.15\%) in rural area. Majority of the study subjects were from urban area with female preponderance and among the relatives, those had abnormal OGTT, majority were from urban residence. A distinguished number of first degree relatives found to have different form of glucose intolerance namely IFG, IGT, IFG+IGT and diabetes which were detected first time. We know prediabetes is a stage of natural history of any form of diabetes. Type 2 diabetes has a strong genetic susceptibility as evident by familial aggregation of this disease for decades, but 
susceptible genetic locus had not been well established now. A study on Chinese population shows abnormal glucose tolerance is common in first-degree relatives of non-insulin-dependent Diabetes Mellitus patients; both insulin resistance and impaired beta cell function are associated with impaired glucose metabolism, which have existed before diagnosis of IFG, IGT and diabetes ${ }^{17}$. Both insulin resistance and impaired b-cell function are important pathophysiologic changes contributing to the onset and development of type 2 diabetes. These and changes in lipid profile have occurred before a patient is diagnosed with abnormal glucose tolerance ${ }^{18}$. No study was found among the first degree relatives of pre-diabetic subjects. In this study all form of glucose intolerance were found among the relatives, signifies that any types of pre diabetes state (IFG, IGT, IFG+IGT) can be the initial abnormal glucose tolerance among the first degree relatives of prediabetic subjects leads to clinical diabetes. Subjects those had abnormal OGTT reports among the total study subjects including both prediabetes and their first degree relatives showed that $66.7 \%$ of them were from urban residence, $14.3 \%$ from semi urban residence and 19\% from rural communities. In this participation from urban area was greatest may be due to more convenience and awareness about diabetes. But most abnormal glucose reports were from urban communities that may be due to diabetogenic life style. Prediabetic subjects are at increased risk of developing diabetes. For primary prevention of type 2 diabetes among them several studies were carried out. Remarkable studies for primary prevention are Diabetes Prevention Program Study, Finnish Diabetes Prevention Study, STOP-NIDDM, TRIPOD, DREAM and XENDOS study ${ }^{19-24}$. Maintenance of ideal body weight, weight reduction if over weight or obese, practice of healthy diet of proper calorie, regular physical exercise are the tools identified as preventive measures. Intensive nutritional and exercise counseling along with or without drugs is the way of prevention. Drugs used in those different studies are Metformin, Rosiglitazone, Troglitazone, Acarbose, Orlistate and Ramipril. Promising result is found. So, from our study first degree relatives of prediabetes are at increase risk of developing any form of glucose intolerance (IFG, IGT, IFG+IGT, diabetes) and the target for prevention of type 2 diabetes.

\section{Conclusion:}

Clustering of pre-diabetes and diabetes is present in families of prediabetic subjects. So they should be taken as a major target for primary prevention of diabetes and prediabetes as like as first degree relatives of type 2 diabetic subjects.

\section{References:}

1. International Diabetes Federation: Diabetes Atlas (2003): $2^{\text {nd }}$ ed. International Diabetes Federation, Brussels, 15-71

2. Abate N, Chandalia M (2001): Ethnicity and type 2 diabetes: Focus on Asian Indians. J Diabetes Complications; 15: 3207

3. Dhawan J, Bray CL, Warburton R, Ghambhir DS, Morris J (1994): Insulin resistance, high prevalence of diabetes, and cardiovascular risk in immigrant Asians. Genetic or environmental effect? Br Heart J; 72: 413-21.

4. Gopalan C (2001): Rising incidence of obesity, coronary heart disease and diabetes in the Indian urban middle class.Possible role of genetic and environmental factors. World Rev Nutr Diet; 90: 127-43.

5. Mohan V (2004): Why Are Indians More Prone to Diabetes? JAPI; 52: 468-474.

6. Radha V, Vimaleswaran KS, Deepa R, Mohan V (2003): The genetics of diabetes mellitus. Indian J Med Res; 117: 225238.

7. Ramachandran A (2005): Epidemiology of Diabetes in India - Three Decades of Research. JAPI; 53: 34-38

8. Ramachandran A, Snehalatha C, Baskar ADS, Mary S, Sathish Kumar CK, Selvam S, Catherine S, Vijay V (2004): Temporal changes in prevalence of diabetes and impaired glucose tolerance associated with lifestyle transition occurring in the rural population in India. Diabetologia; 47: 860-865.

9. Snehalatha C, Ramchandran A, Kapur A, Vijay V (2003): Age-Specific Prevalence and Risk Associations for Impaired Glucose Tolerance in Urban Southern Indian Population. JAPI; 51: 766-769.

10. DECODA Study Group (2003): Age- and Sex-Specific Prevalence of Diabetes and Impaired Glucose Regulation in 11 Asian Cohorts. Diabetes Care; 26: 1770-1780.

11. Sayeed MA, Mahtab H, Khanam PA, Latif ZA, Ali SMK, Banu A, Ahren B, Azad Khan AK (2003): Diabetes and impaired fasting glycemia in a rural population of Bangladesh. Diabetes Care; 26: 1034-1039.

12. Unwin W (2002): Impaired Glucose Tolerance and Impaired Fasting Glycaemia: the current status on definition and intervention. Diabet Med; 19: 708-723.

13. Tony PCY and Cockram CS (2003): The epidemiology of type 2 diabetes. In: Textbook of Diabetes, $3^{\text {rd }}$ ed. John C. Pickup \& Gareth Williams, Eds, Blackwell Science, 6.1-6.14. 
14. Stern MP and Burke JP (2000): Impaired Glucose Tolerance and Impaired Fasting Glucose-Risk Factors or Diagnostic Categories. In: Diabetes Mellitus: A Fundamental and clinical Text, $2^{\text {nd }}$ ed. Derek Leroith, Simeon I. Taylor and Jerrold M. Olefsky, Eds, Lippincott Williams \& Wilkins, 558-595.

15. Balkau B and Eschwege E (2003): The diagnosis and classification of diabetes and impaired glucose regulation. In: Textbook of Diabetes, 3rd ed. John C. Pickup \& Gareth Williams, Eds, Blackwell Science, 2.1-2.13.

16. Hilding A, Eriksson AK, Agardh EE, Grill V, Ahlbom A, Efendic S, Ostenson CG (2006): The impact of family history of diabetes an lifestyle factors on abnormal glucose regulation in middle- aged Swedish men and women. Diabetologia; 49: 2589-98.

17. Han XY, Ji LN, Zhou XH (2004): Insulin sensitivity and beta function in the first-degree relatives of type 2 diabetic patients. Zhonghua Yi Xue Za Zhi; 84: 1777-80.

18. Han XY, Ji LN, Zhou XH (2005): Cross sectional study of the pathophysiology and clinical features in the first-degree relatives of type 2 diabetic patients. Beijing Da Xue Xue Bao; 37: 159-62.

19. Knowler WC, Barrett-Conner E, Fowler SE, Hamman RF, Lachin JM, Walker EA (2002): Reduction in the incidence of type 2 diabetes with lifestyle intervention or metformin. $N$ Engl J Med.; 346: 393-403.

20. Tuomilehto J, Lindström J, Eriksson JG, Valle TT, Hämäläinen H, Ilanne-Parikka P, Keinänen-Kiukaanniemi S, Laakso M,
Louheranta A, Rastas M, Salminen V, Uusitupa M (2001): Prevention of type 2 diabetes mellitus by changes in lifestyle among subjects with glucose tolerance. $N$ Engl J Med; 344: 1343-1350.

21. Chiasson JL, Josse RG, Gomis R, Hanefeld M, Karasik A, laaskso M (the STOP_NIDDM Trial Research group) (2004): Acarbose for the prevention of type 2 diabetes, hypertention and cardiovascular disease in subjects with impaired glucose tolerance: facts and interpretations concerning the critical analysis of the STOP-NIDDM Trial data. Diabetologia; 47: 969-975.

22. Buchanan TA, Xiang AH, Peters RK, Kjos SL, Marroquin A, Goico J, Ochoa C, Tan S, Berkowitz K, Hodis HN, Azen SP (2002): Preservation of pancreatic beta-cell function and prevention of type 2 diabetes by pharmacological treatment of insulin resistance in high-risk Hispanic women. Diabetes; 51: 2796-2803.

23. Gerstein HC, Yusuf S, Bosch J, Pogue J, Sheridan P, Dinccag N, Hanefeld M, Hoogwerf B, Laakso M, Mohan V, Shaw J, Zinman B, Holman RR (2006): Effect of rosiglitazone on the frequency of diabetes in patients with impaired glucose tolerance or impaired fasting glucose: a randomized controlled trial. Lancet; 368: 1096-1105.

24. Torgerson JS, Hauptman J, Boldrin MN, Sjostrom L (2004): XENical in the prevention of diabetes in obese subjects (XENDOS) study: a randomized study of orlistat as an adjunct to lifestyle changes for prevention of type 2 diabetes in obese patients. Diabetes Care; 27: 155-161. 